\title{
Malignant Acanthosis Nigricans with Recurrent Bladder Cancer: A Case Report and Review of Literature
}

This article was published in the following Dove Press journal: OncoTargets and Therapy

\section{Rongxin Zhang \\ Meiya Jiang \\ Wenyi Lei (ID \\ Aoxue Wang}

Department of Dermatology, The Second Hospital of Dalian Medical University, Dalian, Liaoning, II6027, People's Republic of China
Correspondence: Aoxue Wang Department of Dermatology, The Second Hospital of Dalian Medical University,

Dalian, Liaoning, I I6027, People's

Republic of China

Tel +86- 17709875795

$\mathrm{Fax}+86-4 \mathrm{II}-84672130$

Email wangaxdI@I63.com

\begin{abstract}
Acanthosis nigricans (AN) is a rare cutaneous condition that may be a manifestation of certain endocrinological or autoimmune diseases, as well as a potential marker for underlying undiagnosed cancer. AN is classified as either benign or malignant based on its clinical associations. Malignant acanthosis nigricans (MAN), which tends to be extensive and develops rapidly, is considered to be a paraneoplastic syndrome associated with advanced cancer, commonly accompanied by intra-abdominal malignancies. Bladder cancer with AN concomitant with tripe palms (TP) and/or mucosal involvement is relatively rare and, to our knowledge, only seven cases of $\mathrm{AN}$ with bladder cancer have been reported in the English literature. However, to date, there have been no reports of bladder cancer with AN concomitant with TP, skin and mucosal involvement. Here, we present a case of a 71year-old male diagnosed with AN coexisting with recurrent bladder cancer and simultaneous TP and oral AN. In addition, we provide a brief review of the available literature on this topic. Early recognition of skin features associated with internal malignancies provides an opportunity for early diagnosis, treatment of the internal malignancy and monitoring of tumor recurrence.
\end{abstract}

Keywords: malignant acanthosis nigricans, bladder cancer, tripe palms, oral acanthosis nigricans

\section{Introduction}

Acanthosis nigricans (AN) is characterized by thick velvety hyperpigmented plaques of the intertriginous areas, and less commonly, extensor areas of the skin. AN represents a rare cutaneous condition that may be a manifestation of certain endocrinological or autoimmune diseases, and even serve as a potential marker for underlying undiagnosed cancer. ${ }^{1}$ There are two forms of AN, benign or malignant, which are based upon clinical associations. Malignant acanthosis nigricans (MAN) is considered as a paraneoplastic syndrome coexistent with advanced cancer. It is usually associated with intra-abdominal malignancies, with gastric adenocarcinoma being the most common, followed by pancreatic, ovary, lung, esophageal, breast, renal cell and bladder cancers. ${ }^{2}$ MAN lesions mainly appear as hyperpigmented foci which later transform into papillary lesions. MAN tends to be extensive, with rapidly developing changes in the skin often accompanied by mucosal involvement, seborrheic keratoses and tripe palms (TP). ${ }^{1}$ TP, which is generally related to pulmonary and gastric carcinoma, rarely appears on the skin surface of the hands. Due to the similarities in histopathological characteristics with $\mathrm{AN}$, some investigators consider TP as a subtype 
of AN. However, TP is generally more discernable and does not involve the soles. ${ }^{2}$ Moreover, the location and probability of mucosal involvement differ in $\mathrm{AN}$, and hyperpigmentation is often absent on mucosal sites.

Of the two forms of AN, MAN has a lower incidence and is more frequently observed in the middle-aged and elderly. According to the literature, an association of MAN with bladder cancer is relatively rare. Here, we report one such rare case of a patient with mucosal and cutaneous AN and TP along with recurrent bladder cancer. In addition, we present a brief review of the available literature on this topic.

\section{Case Presentation}

A 71-year-old male presented with a history of AN lesions on the hands, feet, fold areas, face and oral cavity that had been present for approximately 4 months. The initial lesions appeared as hyperpigmentation, with a rough and thickened skin surface and mild pruritus, present on the hands, feet, axillary and inguinal regions. Subsequently, lesions also emerged on the face, neck, back of the ears and oral cavity. He was treated with an oral administration of avermectin A and traditional Chinese medicines along with topical steroids and urea vitamin E ointment with no clinical improvement. The skin lesions gradually thickened, developing into wart-like hyperplasias or tumor-like lesions and additional lesions associated with burning sensations surfaced on the buccal mucosa and tongue. According to the patient's medical history, he was initially diagnosed with urinary bladder cancer in 2007. At that time, he received TUR-BT surgery followed by regular administrations of intravesical chemotherapy. In 2013, he was diagnosed with rectal cancer and underwent laparoscopic surgery followed by regular chemotherapy. He was then diagnosed with urinary bladder cancer in 2014, and again underwent TUR-BT surgery followed by intravesical chemotherapy. Additionally, the patient had a history of hypertension for more than 10 years. There was no history of steroid medication use, endocrine abnormalities, hepatitis, other autoimmune diseases or familial hereditary diseases. Family members were unaffected.

Physical examination revealed numerous grey and brown skin papillary papules or verrucous nodules observed in the axillary and inguinal areas, along with diffuse hyperkeratosis and waxy-yellow lesions in the palms and plantar surfaces. Clinical examination of the oral cavity revealed hypertrophic lesions and papillomatosis on the buccal mucosa, gingiva and a glossy tongue without moss (Figure 1A-G). There were no apparent abnormalities in the toenails and hair.

Based on these findings and the exclusion of other diseases, there was a suspicion of AN resulting from malignant tumors. Accordingly, assays for specific serum tumor markers and endocrine measurements were performed, including AFP, CEA, CA125, CA19-9 and CYFRA 21-1, along with an assessment of hypothalamic/pituitary/adrenal
A

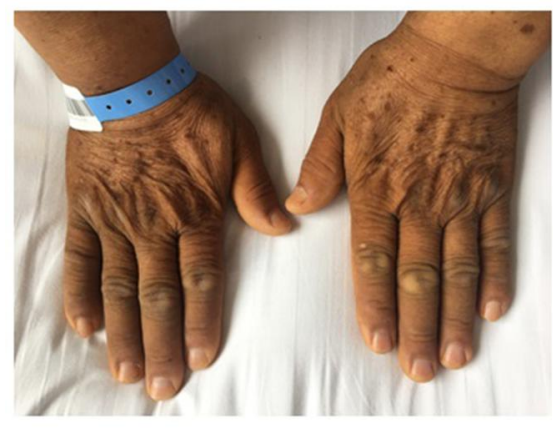

D

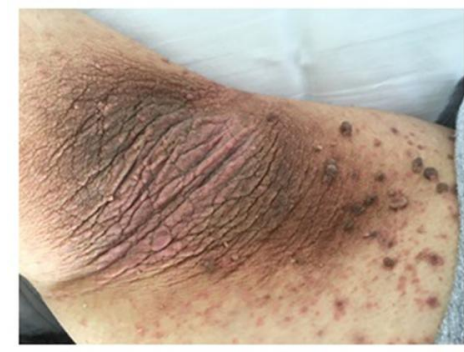

B

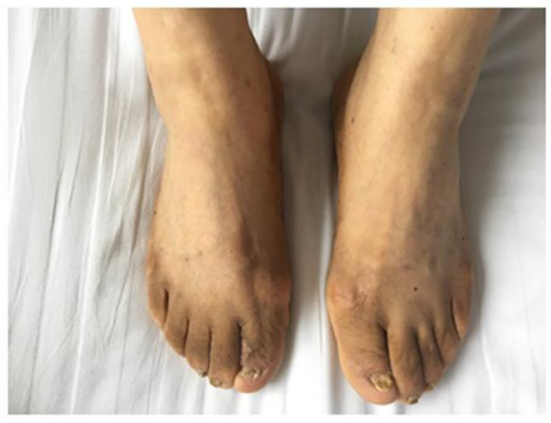

E

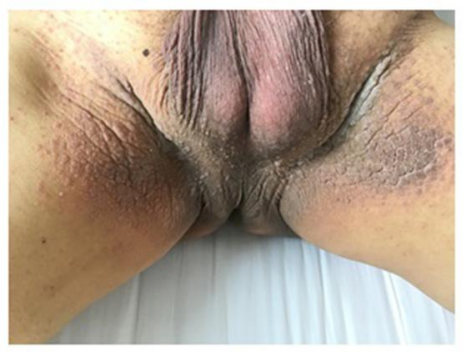

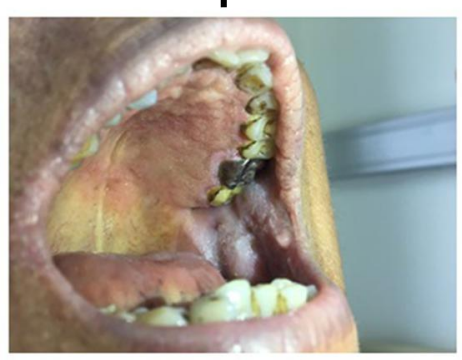

C

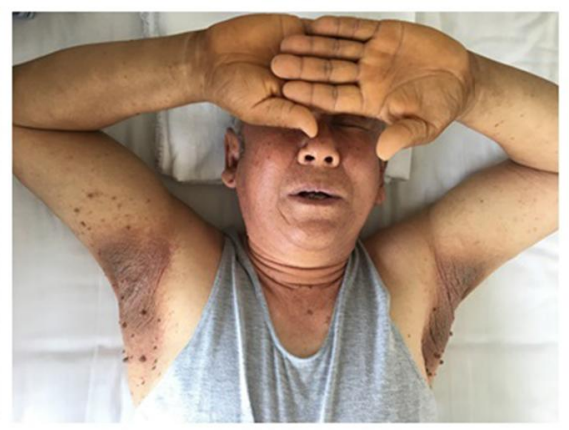

G

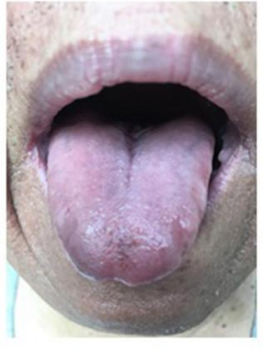

Figure I Acanthosis nigricans on the surfaces of hands (A), feet (B), axilla (C and $\mathbf{D})$, inguinal area (E) and oral cavity (F and $\mathbf{G})$. 
(HPA) gland axis secretory function. Assay results of these tumor markers indicated slight increases in CYFRA21-1 (3.84 ng/mL) and CEA (6.39 ng/mL), with levels of all other tumor markers being within normal limits. Similarly, tests of HPA gland secretory function (such as serum testosterone, thyrotrophic hormone, adrenocorticotropic hormone and cortisol), as well as routine biochemical indicators, were all within normal limits. Ultrasound examination of superficial lymph nodes revealed enlarged lymph nodes in the left inguinal region, with the largest being $35 \mathrm{~mm}$ in diameter. Results from PET-CT imaging showed: 1) that metabolic status of the bladder wall could not be evaluated due to the concentration of urine in the bladder, 2) the presence of multiple hypermetabolic lymph nodes in the paravertebral, retroperitoneal and bilateral iliac vessels, which along with those present in the bilateral pelvic wall and left inguinal region, were considered as sources for metastasis, 3) that the medial tubercle of the left paravertebral erector muscle was also considered as a source for metastasis, and 4) that the diffuse increases in metabolic activity present in the esophagus require further endoscopy examination (Figure 2). Gastroscopy revealed multiple esophageal eminences, with pathological findings being consistent with AN (Figure 3A-D). Pathology examinations of the left inguinal lesion revealed hyperkeratosis of the epidermis, papillomatous hyperplasia and sparse lymphocyte infiltration in the superficial dermis, also consistent with AN (Figure 4). Results from HE-staining of the biopsied left inguinal lymph node revealed that nested masses of cancer cells were present. Immunohistochemical staining for GATA-3, P40, CK7 and CK20 were positive, Villin was partially positive and CDX2 was negative in the cancer cells of the inguinal lymph node. When combining the staining results from the biopsied left inguinal lymph node and the patient's history, the origin of metastasis is considered to be urothelial cancer (Figure 5A-G).

When collating this information on history, skin lesions and pathology, PET-CT and lymph node biopsy, a clinical diagnosis of MAN with recurrent bladder cancer was confirmed. After diagnosis, the patient was transferred to the Oncology Department for systemic chemotherapy. He underwent a chemotherapy regimen consisting of gemcitabine and cisplatin every 3 weeks for 5 cycles. After three cycles, hyperkeratosis of the hands and feet, hyperpigmentation and verrucous hyperplasia in the axillary and inguinal regions and lesions in the oral cavity were all significantly improved (Figure 6A-F). Currently, the patient is receiving immunotherapy with the anti-PD-1

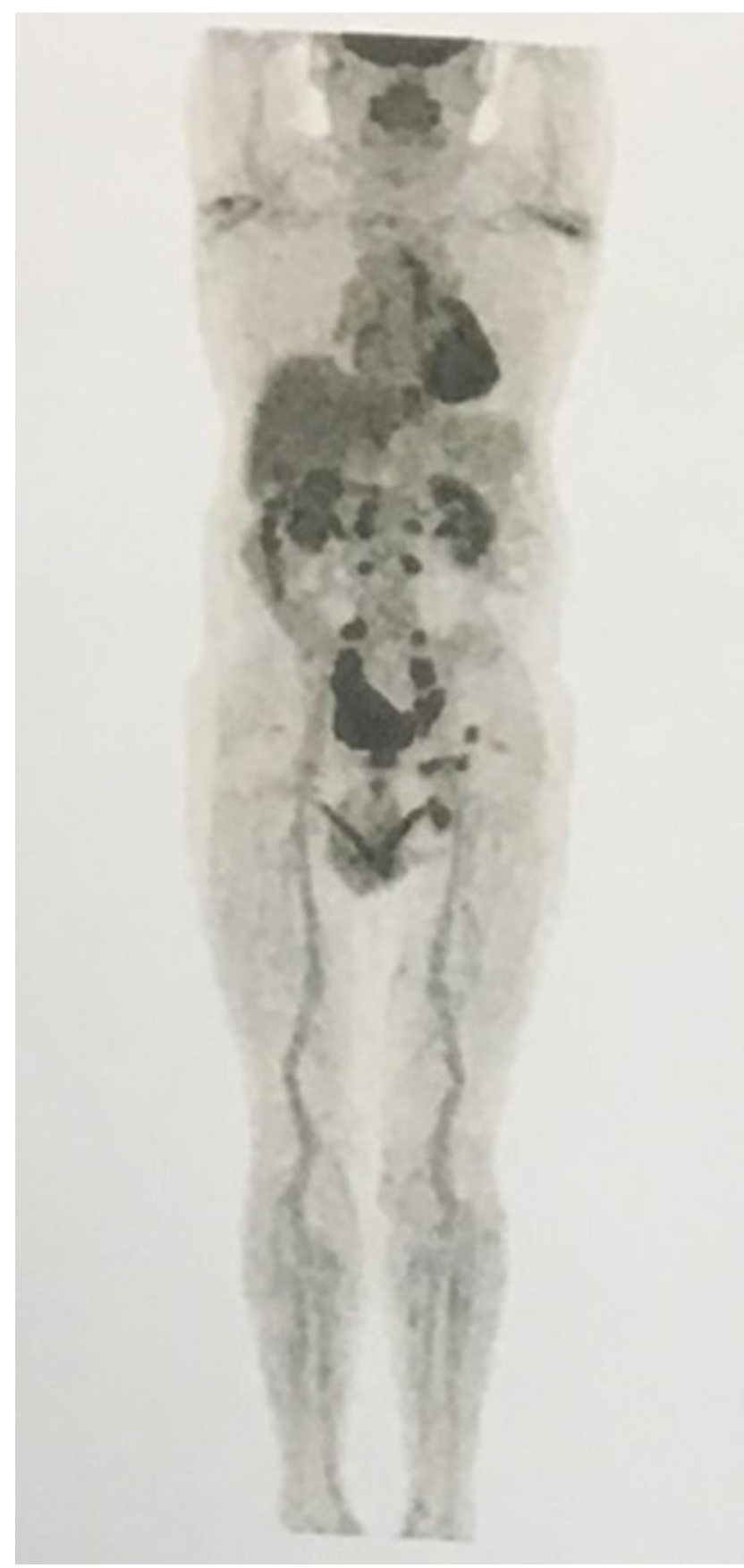

Figure 2 PET-CT results showing multiple hypermetabolic lymph nodes considered metastatic within paravertebral, retroperitoneal, bilateral iliac vessels, bilateral pelvic wall, left inguinal area and medial tubercle of the left paravertebral erector muscle.

monoclonal antibody, tislelizumab, and follow-up in the Oncology Department.

\section{Discussion}

AN, also known as melanokeratosis, was first described by Pollitzer. ${ }^{3}$ It is a rare skin disease characterized by hyperpigmentation, hyperkeratosis, velvety thickening and verrucous 
A
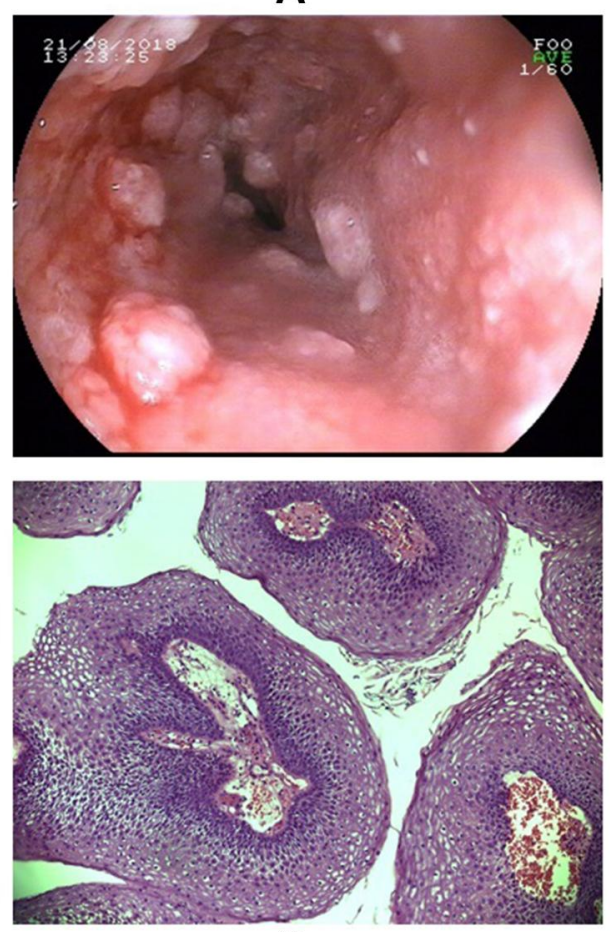

C
B
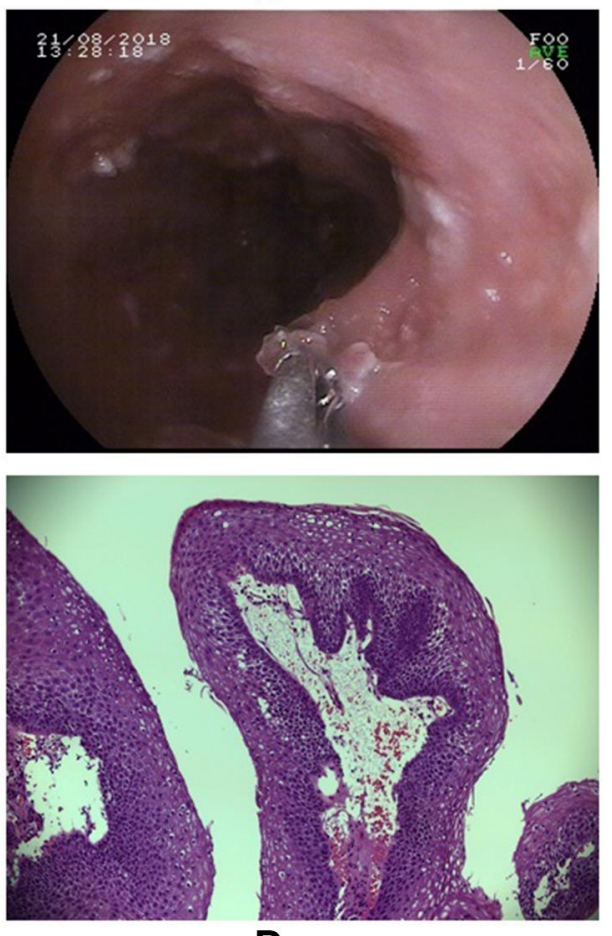

D

Figure 3 Gastroscopy revealing multiple esophageal eminences (A and $\mathbf{B})$, and the pathological findings of esophageal hyperplasia $(\mathbf{C}$ and $\mathbf{D})$ which were consistent with $A N$ $(\mathrm{HE}$ stain $\times 100)$.

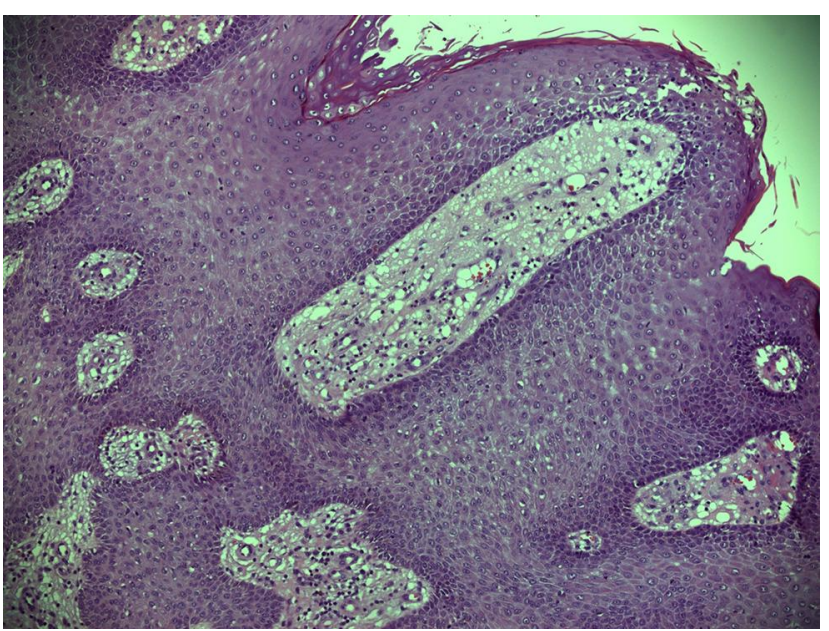

Figure 4 Pathological findings of the left inguinal lesion revealed hyperkeratosis of the epidermis, papillomatous hyperplasia, and sparse lymphocyte infiltration in the superficial dermis (HE stain $\times 100)$.

hyperplasia that can occur on any part of the body, but characteristically affects fold areas, such as axillae, neck, inguinal, antecubital and popliteal surfaces. Clinically, as based upon the description of Schwartz, eight different types of AN have been described: benign, malignant, obesity-associated, syndromic, acral, unilateral, medication- induced and mixed-type. ${ }^{4}$ The main histological features of AN are hyperkeratosis and papillomatosis. ${ }^{2}$ The principal interest in AN resides in its association with malignancy, along with the clinical manifestations of skin lesions and the rapid and potentially lethal nature of the disease. With regard to MAN, the malignant form of $\mathrm{AN}$, this condition is common in the middle-aged and elderly and almost always involves visceral tumors. The most common histological types of MAN-associated tumors involve adenocarcinomas, especially gastric adenocarcinoma, followed by pancreatic, ovary, lung, esophageal, breast, renal cell and bladder cancers. In most cases of MAN (61.3\%), there is a simultaneous diagnosis of cancer. AN occurs prior to cancer diagnosis in approximately $17.6 \%$ of cases and follows a cancer diagnosis in approximately $21.1 \%$ of cases. ${ }^{2,5}$

The development of lesions associated with MAN are rapid, serious and extensive, with the underlying mechanisms for these effects remaining unclear. Results from previous studies have indicated that these effects may be related to the secretion of certain cytokines by malignant tumors. Some examples of these cytokines include tumorderived growth factor, alpha-melanocyte-stimulating factor, peptide substances, platelet-derived growth factor, 

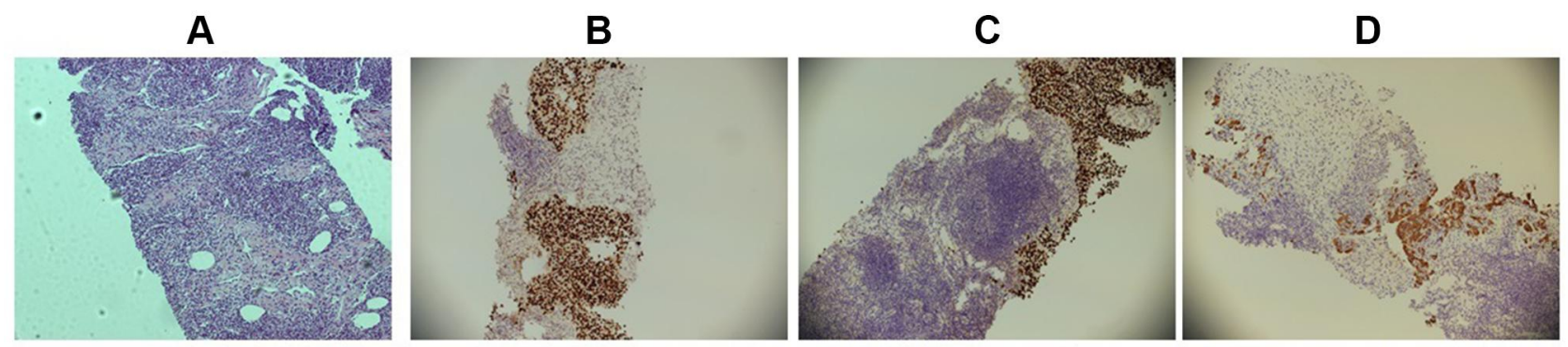

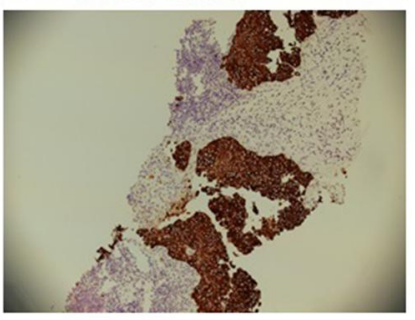

E

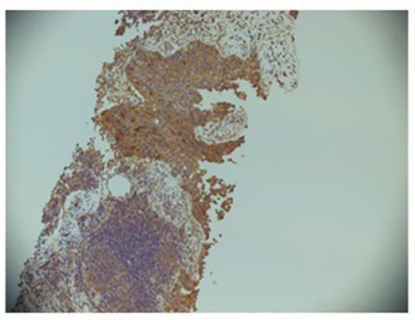

$\mathbf{F}$

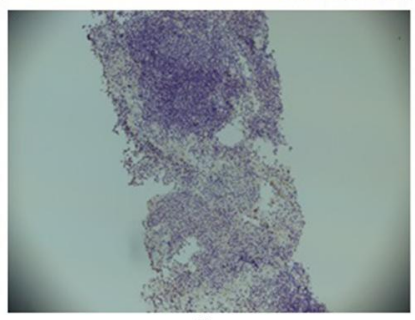

G

Figure 5 Results of left inguinal lymph node biopsy revealed that nested mass cancer cells were present $(\mathbf{A})(\mathrm{HE}$ stain $\times 100)$. Cancer cells in the inguinal lymph node were positive for GATA-3 (B), P40 (C), CK20 (D), CK7 (E), partially positive for Villin (F) and negative for CDX2 (G) (Immunohistochemical stain $\times 100)$.

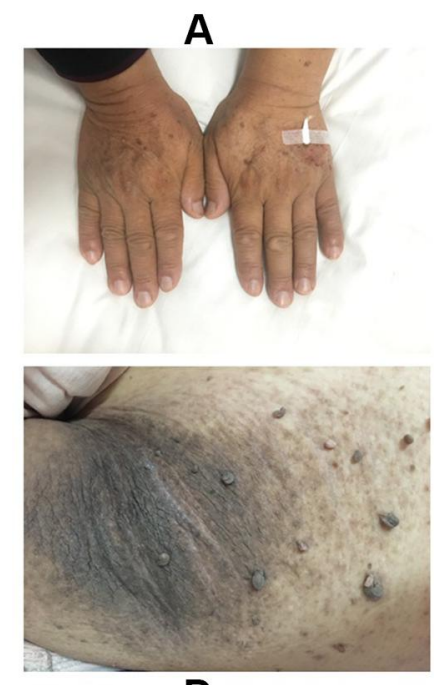

D

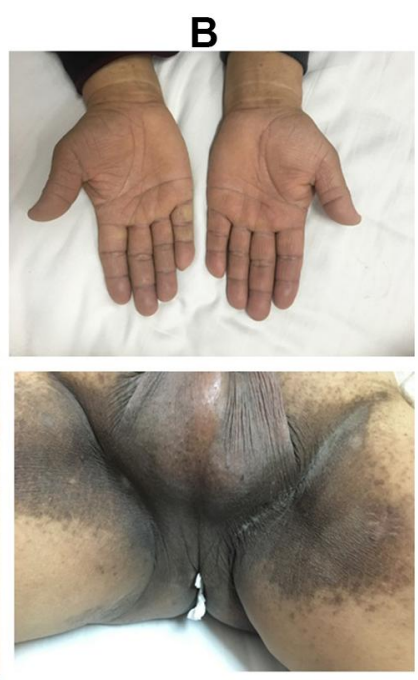

E

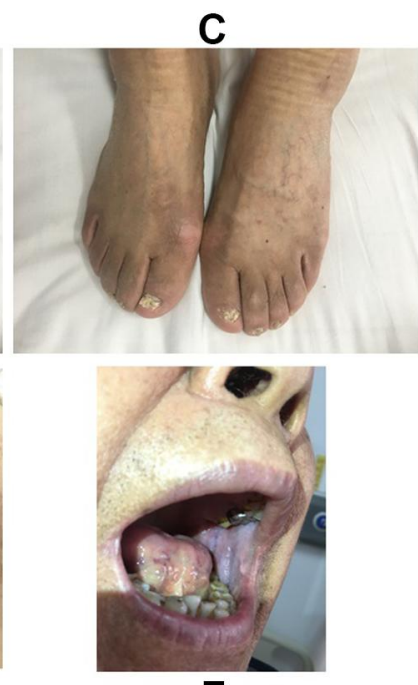

$\mathbf{F}$

Figure 6 After three courses of chemotherapy, hyperkeratosis of the hands and feet (A-C), pigmentation and verrucous hyperplasia on the axillary (D) and inguinal (E) regions and lesions in the oral cavity $(\mathbf{F})$ were all significantly improved.

insulin-like growth factor-1 and, in particular, transforming growth factor-alpha (TGF- $\alpha$ ), whose effects on the epidermal growth factor receptor (EGFR) lead to the proliferation of keratinocytes and dermal fibroblasts. ${ }^{4,5}$ The differentiation of keratinocytes can be partially inhibited with activation of the ERK signaling pathway, which results in a verrucous appearance of the epidermis.

The patient in our case report had a history of malignant tumors from the bladder and rectal cancer that were treated surgically and with a regular follow-up of chemotherapy. However, the AN in this patient occurred suddenly, progressed rapidly and was accompanied by mucosal involvement. Skin and esophageal histopathology were consistent with AN. There were no significant abnormalities in the determination of cortical axis, islet function and blood sugar levels after admission. Due to a high suspicion of malignant tumors in this AN patient, systematic examinations were performed. Multiple enlarged lymph nodes were detected in the left inguinal region by color Doppler ultrasonography and results of PET-CT scans revealed multiple lymph node metastases. A diagnosis of MAN was confirmed based upon the skin 
Table I Summary of Clinical Data on Patients with Bladder Cancer and AN and/or TP and/or Oral AN from 1983 to 2020

\begin{tabular}{|c|c|c|c|c|c|c|c|c|c|c|c|}
\hline \multirow[t]{2}{*}{ Case } & \multirow[t]{2}{*}{ Age } & \multirow[t]{2}{*}{ Gender } & \multicolumn{3}{|c|}{ Clinical Manifestations } & \multicolumn{2}{|c|}{ With Bladder Cancer } & \multicolumn{3}{|c|}{ Tumor Condition } & \multirow[t]{2}{*}{ REF } \\
\hline & & & Skin AN & TP & Oral AN & Prior & Follow & Initial & Recurrent & Metastasis & \\
\hline I & 65 & $\mathrm{~F}$ & $Y$ & $\mathrm{~N}$ & Y & & $Y$ & & Y & Y & [5] \\
\hline 2 & 69 & $\mathrm{~F}$ & $Y$ & $Y$ & $\mathrm{~N}$ & & $Y$ & & $Y$ & $Y$ & [6] \\
\hline 3 & 70 & M & Y & Y & $\mathrm{N}$ & Y & & Y & & Y & [7] \\
\hline 4 & 78 & $\mathrm{~F}$ & $Y$ & $\mathrm{~N}$ & Y & & $Y$ & & $Y$ & & [8] \\
\hline 5 & 73 & M & Y & $\mathrm{N}$ & Y & Y & & Y & & & [9] \\
\hline 6 & 46 & M & $Y$ & $\mathrm{~N}$ & $\mathrm{~N}$ & $Y$ & & $Y$ & & & {$[10]$} \\
\hline 7 & 40 & $F$ & Y & Unk & $\mathrm{N}$ & Y & & Y & & Unk & [II] \\
\hline 8 & 71 & $M$ & $Y$ & $Y$ & $Y$ & & $Y$ & & $Y$ & $Y$ & This case \\
\hline
\end{tabular}

Abbreviations: AN, acanthosis nigricans; TP, tripe palms; F, female; M, male; Y, yes; N, no; Unk, unknown; REF, reference.

lesions, histopathology and PET-CT findings. Results from the biopsy of the left inguinal lymph nodes suggested that the origin of metastases was urothelial carcinoma. Given the past history of two bladder cancers in this patient, we considered the recurrence of bladder cancer metastasis as leading to MAN. While digestive system tumors are common in MAN-associated tumors, clinical reports of bladder cancer with AN are relatively rare. To our knowledge, only seven cases of AN have been reported in the English literature as summarized in Table $1 .^{5-11}$ However, there have been no such cases reported within China. Following this diagnosis, the patient was transferred to the Oncology Department for systematic chemotherapy. After three courses of chemotherapy, the skin condition dramatically improved, likely through blocking the production of cytokines released by the tumors. In this way, AN can serve as a principal cutaneous marker of internal malignancy and as an index to evaluate the condition, efficacy and recurrence of malignancy. We are reminded from this case that MAN, as associated with bladder cancer, should raise the suspicion of its metastasizing process.

Lesions in the oral cavity are more common in patients with MAN. ${ }^{5}$ Extensive papillary lesions of the lip, gingiva, palate and tongue are all characteristics of oral AN, which may then spread to the nose, throat or esophagus. ${ }^{5,12}$ The presence of mucosal involvement in MAN should be regarded as a significant clinical sign due to the high incidence of tumors in patients with mucosal lesions. ${ }^{9}$ In our current case, lesions were not only observed in the buccal mucosa but also the esophagus. The presence of TP represents another potential skin manifestation of a tumor. While tumors with TP and/or mucosal involvement have been reported extensively within the literature, to date, there has been no case report of bladder cancer with AN accompanied with TP, skin and mucosal involvement. The severity of skin lesions in MAN is closely related to the occurrence and development of tumors, and these skin symptoms can significantly improve after the treatment of potential tumors, as was observed in our patient. Although MAN usually has no specific treatment protocol, AN may be treated with retinoic acid or PUVA (psoralen photochemotherapy), with limited effectiveness.

The degree of MAN malignancy is often greater when associated with tumors and, under such conditions, the disease progresses rapidly. Our case as reported here highlights the relationship between the coexistence of skin markers and internal malignancies and thus the need to identify the source of the potential primary malignancy. Early recognition of these skin features provides an opportunity for early diagnosis, treatment of internal malignancies and monitoring of tumor recurrence.

\section{Conclusion}

In summary, MAN represents an extensive and rapidly developing skin condition, which is considered to be a paraneoplastic syndrome associated with advanced cancer. In the present case, MAN was diagnosed based upon typical skin lesions, histopathology and PET-CT findings. While MAN usually has no specific treatment protocol, the MAN in this case improved after chemotherapy. The presence of mucosal involvement and TP in MAN should alert the clinicians to the possibility of a high incidence of tumors. To our knowledge, there have been no case reports in the literature of bladder cancer with AN accompanied with TP along with skin and mucosal involvement. Our case as reported here highlights the relationship between the coexistence of skin markers and internal malignancies and thus the need to identify the source of the potential primary 
malignancy. Early recognition of these skin features provides an opportunity for early diagnosis, treatment of internal malignancies and monitoring of tumor recurrence.

\section{Abbreviations}

AN, acanthosis nigricans; MAN, malignant acanthosis nigricans; TP, tripe palms; TUR-BT, transurethral bladder tumor resection; HPA, hypothalamus pituitary adrenal gland; PET-CT, positron emission tomographycomputed tomography; CEA, carcinoembryonic antigen; TGF- $\alpha$, transforming growth factor-alpha; EGFR, epidermal growth factor receptor; ERK, extracellular regulated protein kinases; PUVA, psoralen photochemotherapy.

\section{Ethical Approval}

The patient's informed consent for publication of the case details including the images was obtained. The study was approved by the Ethics Committee of the Second Hospital of Dalian Medical University.

\section{Acknowledgments}

This work was supported by the Science and Technology Innovation Foundation of Dalian, China (No.2020JJ 27SN073) and the Natural Science Foundation of Liaoning Province, China (No.2020-MS-262).

\section{Disclosure}

The authors declare no conflicts of interest in this work.

\section{References}

1. Patel NU, Roach C, Alinia H, Huang WW, Feldman SR. Current treatment options for acanthosis nigricans. Clin Cosmet Investig Dermatol. 2018;11:407-413. doi:10.2147/CCID.S137527

2. Pentenero M, Carrozzo M, Pagano M, Gandolfo S. Oral acanthosis nigricans, tripe palms and sign of leser-trélat in a patient with gastric adenocarcinoma. Int $J$ Dermatol. 2004;43(7):530-532. doi:10.1111/ j.1365-4632.2004.02159.x.

3. Pollitzer S. Acanthosis nigricans. In: Unna PG, Morris M, Besnier E, editors. Internal Atlas of Rare Skin Diseases. London, UK: Lewis and Co; 1891:1-3.

4. Schwartz RA. Acanthosis nigricans. J Am Acad Dermatol. 1994;31 (1):1-19.

5. Olek-Hrab K, Silny W, Zaba R, Osmola-Mańkowska A, MackiewiczWysocka M, Dańczak-Pazdrowska A. Co-occurrence of acanthosis nigricans and bladder adenocarcinoma-case report. Contemp Oncol (Pozn). 2013;17(3):327-330. doi:10.5114/wo.2013.35287

6. Möhrenschlager M, Vocks E, Wessner DB, Nährig J, Ring J. Tripe palms and malignant acanthosis nigricans: cutaneous signs of imminent metastasis in bladder cancer? J Urol. 2001;165(5):1629-1630. doi:10.1016/s0022-5347(05)66369-0.

7. Singh GK, Sen D, Mulajker DS, Suresh MS. Acanthosis nigricans associated with transitional cell carcinoma of the urinary bladder. Indian J Dermatol. 2011;56(6):722-725. doi:10.4103/0019-5154.91837.

8. Canjuga I, Mravak-Stipetić M, Kopić V, Galić J. Oral acanthosis nigricans: case report and comparison with literature reports. Acta Dermatovenerol Croat. 2008;16(2):91-95.

9. Cairo F, Rubino I, Rotundo R, Prato GP, Ficarra G. Oral Acanthosis nigricans as a marker of internal malignancy. A case report J Periodontol. 2001;72(9):1271-1275. doi:10.1902/jop.2000.72.9.1271.

10. Gohji K, Hasunuma Y, Gotoh A, Shimogaki H, Kamidono S. Acanthosis nigricans associated with transitional cell carcinoma of the urinary bladder. Int $J$ Dermatol. 1994;33(6):433-435. doi:10.1111/j.1365-4362.1994.tb04046.x.

11. Pinto WBVR, Badia BML, Souza PVS, Oliveira ASB, Silva LHL, Farias IB. Paraneoplastic motor neuronopathy and malignant acanthosis nigricans. Arq Neuropsiquiatr. 2019;77(7):527. doi:10. 1590/0004-282X20190076.

12. Ramirez-Amador V, Esquivel-Pedraza L, Caballero-Mendoza E, Berumen-Campos J, Orozco-Topete R, Angeles-Angeles A. Oral manifestations as a hallmark of malignant acanthosis nigricans. J Oral Pathol Med. 1999;28(6):278-281. doi:10.1111/j.1600-0714.1999.tb02039.x

\section{Publish your work in this journal}

OncoTargets and Therapy is an international, peer-reviewed, open access journal focusing on the pathological basis of all cancers, potential targets for therapy and treatment protocols employed to improve the management of cancer patients. The journal also focuses on the impact of management programs and new therapeutic agents and protocols on patient perspectives such as quality of life, adherence and satisfaction. The manuscript management system is completely online and includes a very quick and fair peer-review system, which is all easy to use. Visit http://www.dovepress.com/ testimonials.php to read real quotes from published authors. 